\title{
Brownian Carnot engine
}

\author{
I. A. Martínez ${ }^{1,2 \star \dagger}$, É. Roldán ${ }^{1,3,4 \star \dagger}$, L. Dinis ${ }^{4,5}$, D. Petrov ${ }^{1}$, J. M. R. Parrondo ${ }^{4,5}$ and R. A. Rica ${ }^{1 \star}$
}

The Carnot cycle imposes a fundamental upper limit to the efficiency of a macroscopic motor operating between two thermal baths'. However, this bound needs to be reinterpreted at microscopic scales, where molecular bio-motors ${ }^{2}$ and some artificial micro-engines ${ }^{3-5}$ operate. As described by stochastic thermodynamics ${ }^{6,7}$, energy transfers in microscopic systems are random and thermal fluctuations induce transient decreases of entropy, allowing for possible violations of the Carnot limit ${ }^{8}$. Here we report an experimental realization of a Carnot engine with a single optically trapped Brownian particle as the working substance. We present an exhaustive study of the energetics of the engine and analyse the fluctuations of the finite-time efficiency, showing that the Carnot bound can be surpassed for a small number of non-equilibrium cycles. As its macroscopic counterpart, the energetics of our Carnot device exhibits basic properties that one would expect to observe in any microscopic energy transducer operating with baths at different temperatures ${ }^{9-11}$. Our results characterize the sources of irreversibility in the engine and the statistical properties of the efficiency-an insight that could inspire new strategies in the design of efficient nano-motors.

The Carnot cycle consists of two isothermal processes, where the working substance is respectively in contact with thermal baths at different temperatures $T_{\mathrm{h}}$ and $T_{\mathrm{c}}$, connected by two adiabatic processes, where the substance is isolated and heat is not delivered nor absorbed. An external parameter is changed in such a way that the whole cycle is carried out reversibly. Following this scheme, one could devise a progressing miniaturization of a Carnot engine and eventually reproduce the cycle with a single Brownian particle. In fact, a variety of thermodynamic processes and even a complete Stirling cycle have been already implemented in the mesoscale using micro-manipulation techniques ${ }^{3-5,12-14}$. Interestingly, the exchange of energy between the particle and its surrounding environment becomes stochastic at the microscale and yet one can rigorously define work, heat and efficiency, within the framework of the recently developed stochastic thermodynamics ${ }^{6,7}$.

The experimental realization of a Carnot cycle with a single Brownian particle has remained elusive owing to the difficulties of implementing an adiabatic process. In particular, it is not clear how to isolate a particle from the surrounding fluid ${ }^{15}$. A more feasible strategy is to simultaneously change the temperature and the external parameter keeping constant the Shannon entropy of the particle. However, the necessary fine-tuning of the temperature is an experimental challenge as well. Here we construct a Brownian Carnot engine putting forward an experimental technique that allows precise control of both the effective temperature and the accessible volume of a single microscopic particle (see Methods and refs 16-18). We use a particle with an inherent electric charge and apply a noisy electrostatic force that mimics a thermal bath. In this way, we can achieve temperatures ranging from room temperature (no electrostatic force) up to hundreds or even thousands of kelvins, far above the boiling point of water.

The working substance of our engine is a single optically trapped colloidal particle immersed in water ${ }^{14}$. For small displacements $x$ from the trap equilibrium position, the optical potential is harmonic, $U(x, t)=\kappa x(t)^{2} / 2$, with stiffness $\kappa$. The Hamiltonian or total energy of the particle is $H=\kappa x^{2} / 2+p^{2} /(2 m)$, with $p=m(\mathrm{~d} x / \mathrm{d} t)$ being the linear momentum of the particle and $m$ the mass of the particle. The conjugated force for the external parameter $\kappa$ is $F_{\kappa}(t) \equiv \partial H / \partial \kappa=x^{2}(t) / 2$. As a result, the work necessary to implement a change $\mathrm{d} \kappa$ in the external parameter, $\mathrm{d} W(t)=F_{\kappa}(t) \mathrm{d} \kappa$, and the heat or energy transfer from the thermal bath to the particle, $\mathrm{d} Q(t)=\mathrm{d} H(t)-\mathrm{d} W(t)$, are fluctuating quantities.

The Carnot cycle is implemented by modifying the stiffness $\kappa$ and the environment temperature $T$ (Fig. 1a,b) and consists of two isothermal processes ( $T$ is kept constant and $\kappa$ changes, blue and red curves in Fig. 1b) and two adiabatic processes ( $T$ and $\kappa$ change keeping $T^{2} / \kappa$ constant $^{14}$, green and magenta curves in Fig. 1b). We measure different thermodynamic quantities (temperature, stiffness, heat, work and Shannon entropy, see Methods) under both equilibrium and non-equilibrium driving (Fig. 1b-d). The effective temperature of the particle is obtained from the average potential energy, $T_{\text {part }}(t) \equiv \kappa(t)\left\langle x(t)^{2}\right\rangle / k$, and can differ from the environment temperature $T$ for non-quasistatic protocols. The $T_{\text {part }}-\kappa$ diagram of the engine (Fig. 1b) shows larger fluctuations in the quasistatic equilibrium protocol, because the average is taken over a smaller number of cycles. In the non-equilibrium protocol, the most irreversible steps are the expansions, where the particle remains colder (that is, more confined ${ }^{19}$ ) than the environment. As in a macroscopic gas, the expansion is dominated by an entropic force, namely, the tendency of the gas to fill the available space. In the case of the single Brownian particle, the expansion is driven by thermal fluctuations that allow the particle to move farther away from the centre of the trap. On the other hand, the compression is driven by the trap confining force, which allows the particle to react more rapidly and to follow the equilibrium temperature even in fast cycles in the adiabatic compression. In the isothermal compression, however, we observe a fast initial increase of the temperature of the particle due to the increase of the stiffness. The $F_{\kappa}-\kappa$ diagram (Fig. 1c) resembles the Clapeyron pressure versus volume diagram of a Carnot cycle performed with an ideal gas ${ }^{20}$. The $T_{\text {part }}-S$ diagram of the particle (Fig. 1d) is a rectangle where all of the entropy changes in the system occur in the two isothermal steps. This diagram also gives information about the nature of the irreversibility for a fast driving (open symbols): the effective temperature of the particle in the isothermal processes suggests the presence of an irreversible flow

${ }^{1}$ ICFO-Institut de Ciències Fotòniques, Barcelona Institute of Science and Technology, 08860 Castelldefels (Barcelona), Spain. ${ }^{2}$ Laboratoire de Physique, École Normale Supérieure, CNRS UMR5672 46 Allée d'Italie, 69364 Lyon, France. ${ }^{3}$ Max Planck Institute for the Physics of Complex Systems, Nöthnitzer Str. 38, 01187 Dresden, Germany. ${ }^{4}$ GISC-Grupo Interdisciplinar de Sistemas Complejos, 28040 Madrid, Spain. ${ }^{5}$ Departamento de Fisica Atómica, Molecular y Nuclear, Universidad Complutense Madrid, 28040 Madrid, Spain. These authors contributed equally to this work. *e-mail: martinez.ignacio@ens-lyon.fr; edgar@pks.mpg.de; rul@ugr.es 

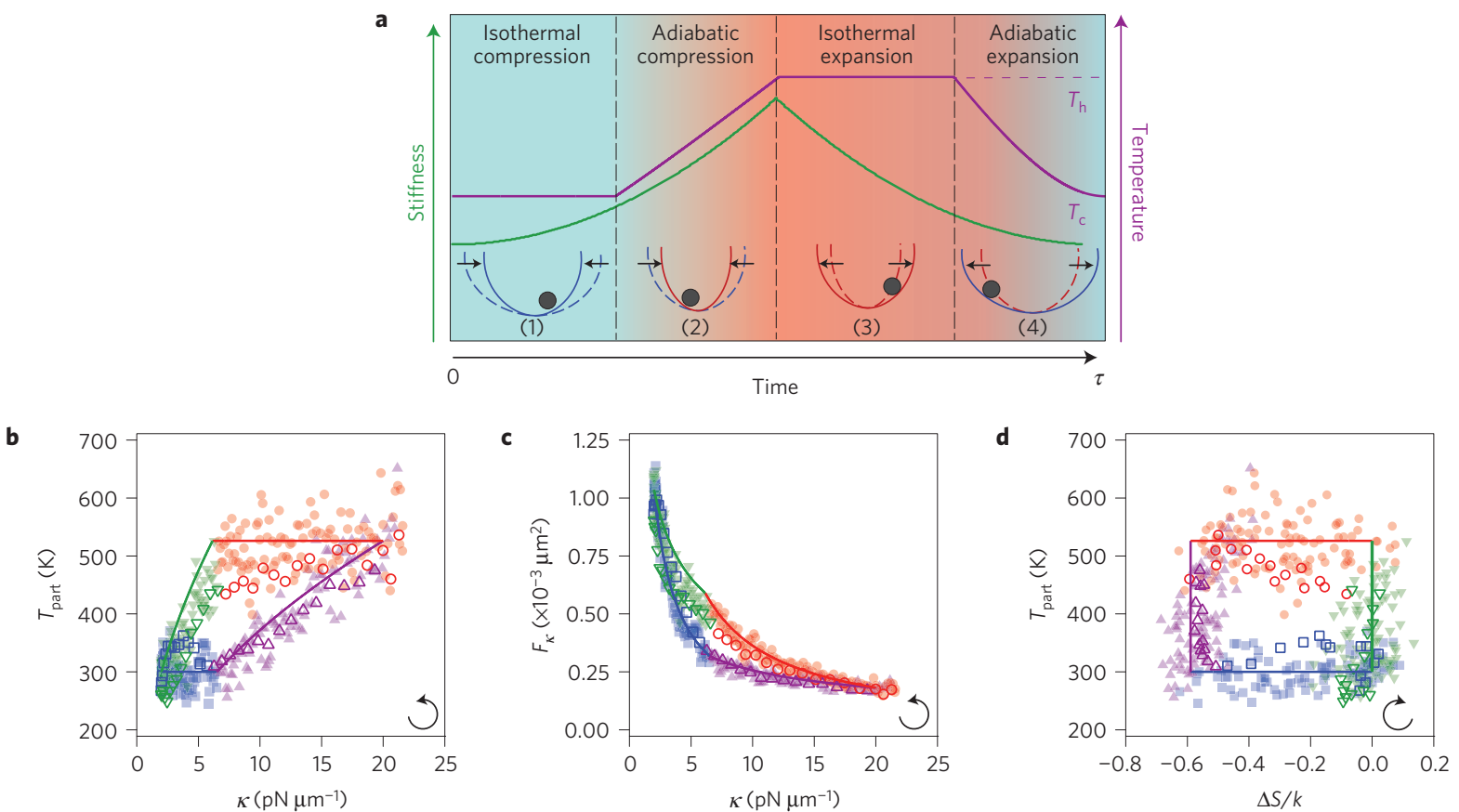

Figure 1 | The Brownian Carnot engine. a, Time evolution of the experimental protocol. b-d, Thermodynamic diagrams of the engine: (1) isothermal compression (blue); (2) adiabatic compression (magenta); (3) isothermal expansion (red); (4) adiabatic expansion (green). Solid lines are the analytical values in the quasistatic limit. Filled symbols are obtained from ensemble averages over cycles of duration $\tau=200$ ms; open symbols are obtained for $\tau=30 \mathrm{~ms}$. The black arrow indicates the direction of the operation of the engine. $\mathbf{b}, T_{\text {part }}-\kappa$ diagram. $\mathbf{c}$, Clapeyron diagram. The area within the cycle is equal to the mean work obtained during the cycle. d, $T_{\text {part }}-S$ diagram. The entropy changes only in the isothermal steps.

of energy between the reservoir and the particle, resembling the endo-reversible engine introduced by Curzon and Ahlborn ${ }^{21,22}$.

During a cycle of duration $\tau$, the working substance of the engine exchanges heat with the different thermal baths it is put in contact with, and under appropriate conditions it is able to extract work. We call $W_{\tau}$ and $Q_{\tau}$ the work exerted on the particle and the heat transferred from the environment to the particle along a cycle, respectively. The exchanged heat equals $Q_{\tau}=\Delta H_{\tau}-W_{\tau}$. Both work and heat along the whole cycle (Fig. $2 \mathrm{a}$ ) converge to their quasistatic averages $\left\langle\cdot{ }_{\infty}\right\rangle$ following $\left\langle W_{\tau}\right\rangle=\left\langle W_{\infty}\right\rangle+\Sigma_{\text {ss }} / \tau$ (ref. 23). Here, $\left\langle W_{\infty}\right\rangle$ is the quasistatic value of the work done per cycle and the term $\Sigma_{\text {ss }} / \tau$ accounts for the (positive) dissipation, which decays to zero like $1 / \tau$ (ref. 24). In the case of the average heat per cycle, $\left\langle Q_{\tau}\right\rangle$, we find that the dissipative term is negative, that is, $\left\langle Q_{\tau}\right\rangle=\left\langle Q_{\infty}\right\rangle-\Sigma_{\text {ss }} / \tau$ with $\Sigma_{\mathrm{ss}}>0$.

To quantify the performance of the engine, we analyse its power output and efficiency. First, we measure the power output as the mean total work exchanged during a cycle divided by the total duration of the cycle (Fig. $2 \mathrm{~b}$ ), $P_{\tau}=-\left\langle W_{\tau}\right\rangle / \tau$. For $\tau=10 \mathrm{~ms},\left\langle W_{\tau}\right\rangle$ is positive, the particle behaves as a heat pump and the power is negative. For larger values of $\tau$ the power increases, becoming positive, and eventually reaches a maximum value $P_{\max }=6.34 k T_{\mathrm{c}} \mathrm{s}^{-1}$. Above that maximum, $P_{\tau}$ decreases monotonically when increasing the cycle length. The data of $P_{\tau}$ versus $\tau$ fit well to the expected law $P_{\tau}=-\left(\left\langle W_{\infty}\right\rangle+\Sigma_{\text {ss }} / \tau\right) / \tau$. The efficiency is given by the ratio between the extracted work and the input of heat, which is usually considered as the heat flowing from the hot thermal bath to the system. In our experiment, however, there is a non-zero fluctuating heat in the adiabatic steps, which must be taken into account in the definition of the stochastic efficiency of the engine during a finite number of cycles. Here we will consider this heat as input (see Methods for alternative definitions of the efficiency). We define $W_{\tau}^{(i)}$ as the sum of the total work exerted on the particle along $i \geq 1$ cycles of duration $\tau$, and $Q_{\alpha, \tau}^{(i)}$ as the sum over $i$ cycles of the heat transferred to the particle in the $\alpha$ th subprocess $(\alpha=1,2,3,4$, see Fig. 1). We therefore introduce the following definition of stochastic efficiency:

$$
\eta_{\tau}^{(i)}=\frac{-W_{\tau}^{(i)}}{Q_{2, \tau}^{(i)}+Q_{3, \tau}^{(i)}+Q_{4, \tau}^{(i)}}
$$

The long-term efficiency of the motor is given by $\eta_{\tau} \equiv \eta_{\tau}^{(i)}$ with $i \rightarrow \infty$. In the quasistatic limit, the average heat in the adiabatic processes vanishes yielding $\eta_{\infty}=\eta_{\mathrm{C}} \equiv 1-T_{\mathrm{c}} / T_{\mathrm{h}} \simeq 0.43$ (Fig. 2b). Moreover, the standard efficiency at maximum power, $\eta^{*} \simeq(0.25 \pm 0.05)$, is in agreement with the Curzon-Ahlborn expression for finite-time cycles $\eta_{\mathrm{CA}}=1-\sqrt{T_{\mathrm{c}} / T_{\mathrm{h}}} \simeq 0.25$ (refs 21,25 ).

Very recently, much attention has been drawn to the statistical properties of the efficiency of stochastic engines. Using fluctuation theorems, it was shown that the probability density function (PDF) of the efficiency of an autonomous or symmetrically driven engine has a local minimum precisely at the Carnot value $\eta_{\mathrm{C}}$ (ref. 26). For non-symmetric driving protocols, such as our Carnot cycle, there are several theoretical predictions concerning the PDF as well as the large deviation function of the stochastic efficiency ${ }^{10,11}$. To test some of these predictions, we measure the PDF $\rho_{\tau, i}(\eta)$ of the stochastic efficiency $\eta_{\tau}^{(i)}$ (Methods). Close to equilibrium, near the maximum power output of the engine, the distribution is bimodal when summing over several cycles ${ }^{9,11}$ (Fig. 3). Indeed, local maxima of $\rho_{\tau, i}(\eta)$ appear above standard efficiency for large values of $i$. Another universal feature tested here is that the tails of the distribution follow a power law, $\rho_{\tau, i}(\eta \rightarrow \pm \infty) \sim \eta^{-2}$ (inset of Fig. 3 $)^{11,27}$. In the Supplementary Information, we discuss in detail and provide further experimental tests of other universal properties of the PDF and the large deviation function of the stochastic efficiency.

We have realized the first Brownian Carnot engine with a single microscopic particle as a working substance that is able to transform the heat transferred from thermal fluctuations into mechanical work, characterizing both its mean behaviour and fluctuations. At 
a

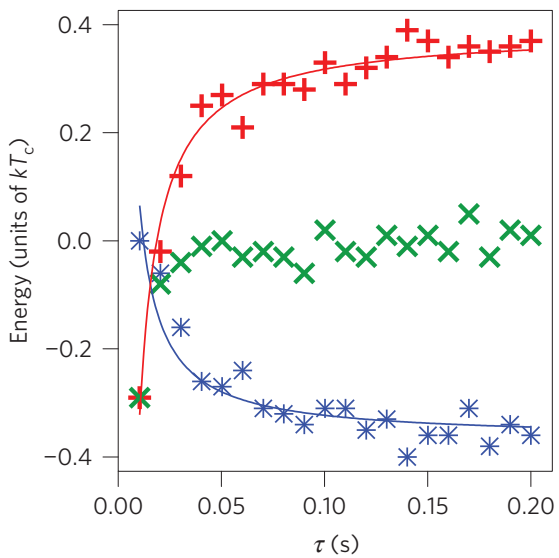

b

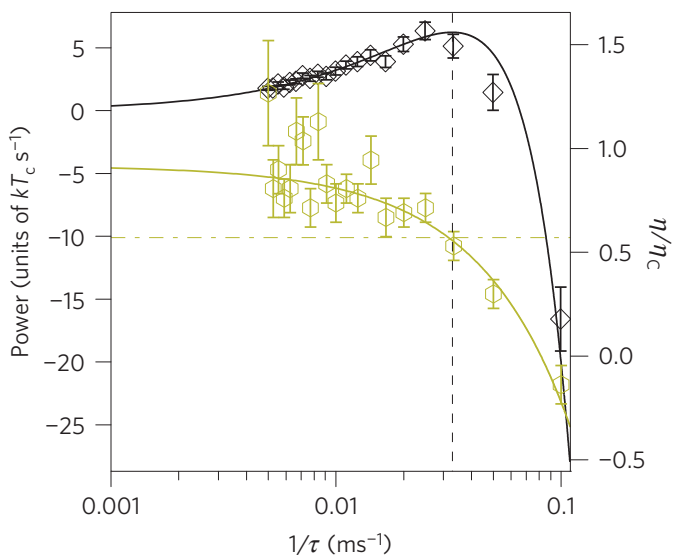

Figure 2 | Energetics of the Brownian Carnot engine. a, Ensemble averages of stochastic work $\left(\left\langle W_{\tau}\right\rangle\right.$, blue stars) and heat ( $\left\langle Q_{\tau}\right\rangle$, red pluses) transferred in one cycle as a function of the cycle duration. Green crosses are the average total energy change of the working substance $\left\langle\Delta H_{\tau}\right\rangle$. Thin lines are fits to $A+B / \tau$. b , Power output $P_{\tau}=-\left\langle W_{\tau}\right\rangle / \tau$ (black diamonds, left axis) and long-term efficiency $\eta_{\tau}$ (yellow hexagons, right axis) as a function of the inverse of the cycle time. The black curve is a fit $P_{\tau}=\left(\left\langle W_{\infty}\right\rangle+\Sigma_{\mathrm{ss}} / \tau\right) / \tau$, yielding $\left\langle W_{\infty}\right\rangle=(-0.38 \pm 0.01) \mathrm{k} T_{\mathrm{c}}$ and $\Sigma_{\mathrm{ss}}=(5.7 \pm 0.3) \mathrm{k} T_{\mathrm{c}}$ ms with a reduced chi-square of $\chi_{\text {red }}^{2}=1.08$. The solid yellow line is a fit to $\eta_{\tau}=\left(\eta_{C}+\tau_{W} / \tau\right) /\left(1+\tau_{Q} / \tau\right)$, which yields $\eta_{\infty}=(0.92 \pm 0.06) \eta_{C}, \tau_{W}=(-11 \pm 2) \mathrm{ms}, \tau_{Q}=(-0.6 \pm 6.0) \mathrm{ms}$ with $\chi_{\text {red }}^{2}=0.76$. Yellow dash-dot line is the Curzon-Ahlborn efficiency $\eta_{C A}=1-\sqrt{T_{c} / T_{h}}=0.25=0.57 \eta c$, which is in excellent agreement with the location of the maximum power (vertical black dashed line). Ensemble averages are done over $50 \mathrm{~s}$ and error bars are obtained with a statistical significance of $90 \%$.

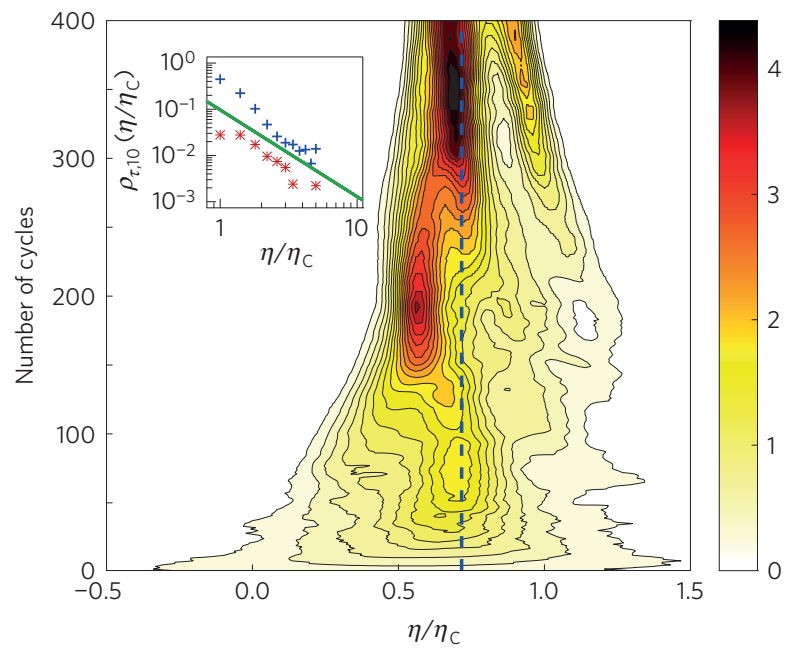

Figure 3 | Efficiency fluctuations at maximum power. Contour plot of the PDF of the efficiency $\rho_{\tau=40 \mathrm{~ms}, i}(\eta)$ computed summing over $i=1$ to 400 cycles (left axis). The long-term efficiency (averaged over $\tau_{\exp }=50 \mathrm{~s}$ ) is shown with a vertical blue dashed line. Super Carnot efficiencies appear even far from quasistatic driving. Inset: tails of the distribution for $\rho_{\tau=40 \mathrm{~ms}, 10}(\eta)$ (blue pluses, positive tail; red stars, negative tail). The green line is a fit to a power law to all the data shown, whose exponent is $\gamma=(-1.9 \pm 0.3)$.

slow driving, our engine attains the fundamental limit of Carnot efficiency. The maximum power performed by our engine is $\sim 250$ larger than that of previous micro-engines ${ }^{3}$ and only one order of magnitude below the power developed by some biological molecular motors such as myosin ${ }^{2}$. Our results could be exploited in the design of new biologically inspired nano-engines ${ }^{28}$ or artificial nanorobots ${ }^{29}$. In vacuum, trapping techniques could benefit from our study of the efficiency fluctuations to build engines capable of outperforming Carnot efficiency ${ }^{30-32}$.

\section{Methods}

Methods and any associated references are available in the online version of the paper.
Received 22 February 2015; accepted 16 September 2015; published online 26 October 2015

\section{References}

1. Carnot, S. Annales scientifiques de l'École Normale Supérieure Vol. 1, 393-457 (Société mathématique de France, 1872).

2. Howard, J. Mechanics of Motor Proteins and The Cytoskeleton (Sinauer Associates Sunderland, 2001).

3. Blickle, V. \& Bechinger, C. Realization of a micrometre-sized stochastic heat engine. Nature Phys. 8, 143-146 (2012).

4. Roldán, E., Martínez, I. A., Parrondo, J. M. R. \& Petrov, D. Universal features in the energetics of symmetry breaking. Nature Phys. 10, 457-461 (2014).

5. Koski, J. V., Maisi, V. F., Pekola, J. P. \& Averin, D. V. Experimental realization of a szilard engine with a single electron. Proc. Natl Acad. Sci. USA 111, 13786-13789 (2014).

6. Sekimoto, K. Lecture Notes in Physics Vol. 799 (Springer Verlag, 2010).

7. Seifert, U. Stochastic thermodynamics, fluctuation theorems and molecular machines. Rep. Prog. Phys. 75, 126001 (2012).

8. Wang, G. M., Sevick, E. M., Mittag, E., Searles, D. J. \& Evans, D. J. Experimental demonstration of violations of the second law of thermodynamics for small systems and short time scales. Phys. Rev. Lett. 89, 050601 (2002).

9. Polettini, M., Verley, G. \& Esposito, M. Efficiency statistics at all times: Carnot limit at finite power. Phys. Rev. Lett. 114, 050601 (2015).

10. Verley, G., Willaert, T., Van den Broeck, C. \& Esposito, M. Universal theory of efficiency fluctuations. Phys. Rev. E 90, 052145 (2014).

11. Gingrich, T. R., Rotskoff, G. M., Vaikuntanathan, S. \& Geissler, P. L. Efficiency and large deviations in time-asymmetric stochastic heat engines. New J. Phys. 16, 102003 (2014).

12. Toyabe, S., Sagawa, T., Ueda, M., Muneyuki, E. \& Sano, M. Experimental demonstration of information-to-energy conversion and validation of the generalized jarzynski equality. Nature Phys. 6, 988-992 (2010).

13. Roldán, E., Martínez, I. A., Dinis, L. \& Rica, R. A. Measuring kinetic energy changes in the mesoscale with low acquisition rates. Appl. Phys. Lett. 104, 234103 (2014).

14. Martínez, I. A., Roldán, É, Dinis, L., Petrov, D. \& Rica, R. A. Adiabatic processes realized with a trapped Brownian particle. Phys. Rev. Lett. 114, 120601 (2015)

15. Crooks, G. E. \& Jarzynski, C. Work distribution for the adiabatic compression of a dilute and interacting classical gas. Phys. Rev. E 75, 021116 (2007)

16. Martínez, I. A., Roldán, É, Parrondo, J. M. R. \& Petrov, D. Effective heating to several thousand kelvins of an optically trapped sphere in a liquid. Phys. Rev. E 87, 032159 (2013).

17. Mestres, P., Martinez, I. A., Ortiz-Ambriz, A., Rica, R. A. \& Roldan, E. Realization of nonequilibrium thermodynamic processes using external colored noise. Phys. Rev. E 90, 032116 (2014). 
18. Bérut, A., Petrosyan, A. \& Ciliberto, S. Energy flow between two hydrodynamically coupled particles kept at different effective temperatures. Europhys. Lett. 107, 60004 (2014).

19. Gieseler, J., Deutsch, B., Quidant, R. \& Novotny, L. Subkelvin parametric feedback cooling of a laser-trapped nanoparticle. Phys. Rev. Lett. 109, 103603 (2012)

20. Feynman, R., Leighton, R. \& Sands, M. The Feynman Lectures on Physics 2nd edn, Vol. 1 (Addison-Wesley, 1963).

21. Curzon, F. \& Ahlborn, B. Efficiency of a Carnot engine at maximum power output. Am. J. Phys. 43, 22-24 (1975).

22. Ouerdane, H., Apertet, Y., Goupil, C. \& Lecoeur, P. Continuity and boundary conditions in thermodynamics: From Carnot's efficiency to efficiencies at maximum power. Eur. Phys. J. Spec. Top. 224, 839-864 (2015).

23. Sekimoto, K. \& Sasa, S.-i. Complementarity relation for irreversible process derived from stochastic energetics. J. Phys. Soc. Jpn 66, 3326-3328 (1997).

24. Bonanca, M. V. S. \& Deffner, S. Optimal driving of isothermal processes close to equilibrium. J. Chem. Phys. 140, 244119 (2014).

25. Esposito, M., Kawai, R., Lindenberg, K. \& Van den Broeck, C. Efficiency at maximum power of low-dissipation Carnot engines. Phys. Rev. Lett. 105, 150603 (2010).

26. Verley, G., Esposito, M., Willaert, T. \& den Broeck, C. V. The unlikely Carnot efficiency. Nature Commun. 5, 5721 (2014).

27. Proesmans, K., Cleuren, B. \& Van den Broeck, C. Stochastic efficiency for effusion as a thermal engine. Europhys. Lett. 109, 20004 (2015).

28. Sarikaya, M., Tamerler, C., Jen, A. K.-Y., Schulten, K. \& Baneyx, F. Molecular biomimetics: Nanotechnology through biology. Nature Mater. 2, 577-585 (2003).

29. Douglas, S. M., Bachelet, I. \& Church, G. M. A logic-gated nanorobot for targeted transport of molecular payloads. Science 335, 831-834 (2012).

30. Roßnagel, J., Abah, O., Schmidt-Kaler, F., Singer, K. \& Lutz, E. Nanoscale heat engine beyond the Carnot limit. Phys. Rev. Lett. 112, 030602 (2014).
31. Gieseler, J., Quidant, R., Dellago, C. \& Novotny, L. Dynamic relaxation of a levitated nanoparticle from a non-equilibrium steady state. Nature Nanotech. 9, 358-364 (2014)

32. Millen, J., Deesuwan, T., Barker, P. \& Anders, J. Nanoscale temperature measurements using non-equilibrium Brownian dynamics of a levitated nanosphere. Nature Nanotech. 9, 425-429 (2014).

\section{Acknowledgements}

I.A.M., É.R., D.P. and R.A.R. acknowledge financial support from Fundació Privada Cellex Barcelona. I.A.M., D.P. and R.A.R. acknowledge financial support from gran NanoMQ (FIS2011-24409, MINECO). I.A.M. acknowledges financial support from the European Research Council Grant OUTEFLUCOP. É.R., L.D. and J.M.R.P. acknowledge financial support from grant ENFASIS (FIS2011-22644, MINECO) and TerMic (FIS2014-52486-R, MINECO). We wish to acknowledge the work of S. Corcuff at the earliest stage of the project and fruitful discussions with R. Brito. D. Petrov passed away on 3 February 2014. He initiated the development of this project while he was the leader of the Optical Tweezers group at ICFO. We mourn the loss of a great colleague and friend.

\section{Author contributions}

I.A.M. designed the experiment, obtained all experimental data and analysed experimental data. É.R. designed the experiment, analysed experimental data and supported theoretical aspects. L.D. supported theoretical aspects. D.P. proposed and established the project, and supervised the experiment. J.M.R.P. proposed and established the project and developed its theoretical aspects. R.A.R. supported and supervised the experiment. All authors discussed the results and wrote the manuscript.

\section{Additional information}

Supplementary information is available in the online version of the paper. Reprints and permissions information is available online at www.nature.com/reprints. Correspondence and requests for materials should be addressed to I.A.M., É.R. or R.A.R.

\section{Competing financial interests}

The authors declare no competing financial interests. 


\section{Methods}

Experimental set-up. Polystyrene microspheres of diameter $1 \mu \mathrm{m}$

(G. Kisker-Products for Biotechnology) are diluted in deionized and filtered water to a final concentration of a few spheres per millilitre. The spheres are inserted into a custom-made electrophoretic fluid chamber with two electrodes. A Gaussian white noise signal is generated with an independent generator (Tabor electronics, WW1071). The external noise is modulated by a custom-made voltage multiplier (100 kHz bandwidth) with a signal $\left(V_{\mathrm{T}}\right)$ generated by a dual generator (Tabor electronics, dual channel WW5062). The output signal of the multiplier is amplified 1,000 times with a high-voltage power amplifier (TREK, 623B) before being applied to the electrodes.

The optical potential is generated by a $980 \mathrm{~nm}$ laser beam which is inserted through an oil immersion objective (Nikon, CFI PL FL $\times 100$ NA 1.30) into the fluid chamber. The detection of the motion of the particle is achieved by an additional $532 \mathrm{~nm}$ laser beam that is passed through the trapping objective. The forward scattered light is collected by an additional microscope objective $(\times 10, \mathrm{NA}=0.10)$, and its back focal-plane field distribution is analysed by a quadrant position detector (New Focus 2911) at an acquisition rate of $2 \mathrm{kHz}$.

A laser controller (Arroyo Instruments 4210) allows the management of the optical power at a maximum rate of $250 \mathrm{kHz}$ using an external voltage $V_{\kappa}$. The trap stiffness depends linearly on the optical power and can be controlled at the same rate as it. The signal sent to the laser controller $\left(V_{\kappa}\right)$ is also generated by the dual generator, and is hence synchronized with $V_{\mathrm{T}}$.

Experimental protocol. The electronic control of the protocol allows us to implement it at different cycle times without loss of resolution, ranging from $\tau=10 \mathrm{~ms}$ to $\tau=200 \mathrm{~ms}$, during $\tau_{\text {exp }}=50 \mathrm{~s}$. For simplicity, we impose a time-symmetric protocol for the stiffness, $\{\kappa(t)\}_{t=0}^{\tau}$ with $\kappa(t)=\kappa(\tau-t)$. The stiffness increases quadratically with time from $t=0$ to $t=\tau / 2$ and decreases at the same rate from $\tau / 2$ to $\tau$. We fix the minimum and maximum values of the stiffness, $\kappa_{\mathrm{I}}=\kappa(0)=(2.0 \pm 0.2) \mathrm{pN} \mu \mathrm{m}^{-1}$ and $\kappa_{\mathrm{III}}=\kappa(\tau / 2)=(20.0 \pm 0.2) \mathrm{pN} \mathrm{\mu m}^{-1}$ respectively. For convenience, we define $\kappa_{\mathrm{II}}=\kappa(\tau / 4)=(6.5 \pm 0.2) \mathrm{pN} \mu \mathrm{m}^{-1}$. The geometry of the Carnot cycle imposes the value of $\kappa_{\mathrm{IV}}=\left(\kappa_{\mathrm{III}} / \kappa_{\mathrm{II}}\right) \kappa_{\mathrm{I}}=\kappa\left(\tau^{*}\right)=(6.2 \pm 0.2) \mathrm{pN} \mu \mathrm{m}^{-1}$, where $\tau^{*}$ yields $\tau^{*}=\tau-(\tau / 2) \sqrt{\left(\kappa_{\mathrm{III}} / \kappa_{\mathrm{II}}\right)-1 /\left(\kappa_{\mathrm{III}} / \kappa_{\mathrm{I}}\right)-1} \simeq 0.76 \tau$. The temperature of the particle remains constant at the isothermal steps: $T_{\mathrm{I}}=T_{\mathrm{II}}=T_{\mathrm{c}}=300 \mathrm{~K}$ during $t \in[0, \tau / 2]$ and $T_{\mathrm{III}}=T_{\mathrm{IV}}=T_{\mathrm{h}}=525 \mathrm{~K}$ during $t \in\left[\tau / 2, \tau^{*}\right]$. Along the adiabatic steps, the temperature changes smoothly while $T^{2}(t) / \kappa(t)$ remains constant to ensure that the total Shannon entropy of the system is conserved ${ }^{14,33}$.

Data analysis. For each cycle of duration $\tau$, we sample the position of the bead with respect to the centre along the $x$ axis set by the direction of the external field. In all cases, we measure trajectories of the position $\left\{x_{t}\right\}_{t=0}^{\tau}$ with sampling rate $2 \mathrm{kHz}$ ( $\Delta t=5 \mathrm{~ms}$ ) along $\tau_{\exp }=50 \mathrm{~s}$. The stochastic work exerted to the particle in the interval of time $[t, t+\Delta t]$ in a single realization is given by

$$
\begin{aligned}
\mathrm{d} W_{\mathrm{t}} & =F_{\kappa}\left(x_{\mathrm{t}}, t\right) \circ \mathrm{d} \kappa_{\mathrm{t}} \\
& =\frac{F_{\kappa}\left(x_{\mathrm{t}}, t\right)+F_{\kappa}\left(x_{\mathrm{t}+\Delta t}, t+\Delta t\right)}{2} \cdot\left[\kappa_{\mathrm{t}+\Delta t}-\kappa_{\mathrm{t}}\right]
\end{aligned}
$$

where o denotes the Stratonovich product (ref. 6) and $F_{K}\left(x_{\mathrm{t}}, t\right)=\partial U\left(x_{\mathrm{t}}, t\right) / \partial \kappa_{\mathrm{t}}=x_{\mathrm{t}}^{2} / 2$ is the generalized force conjugated to the control parameter $\kappa$. The stochastic heat transferred in $[t, t+\Delta t]$ from the effective thermal bath to the particle is calculated using the first law of thermodynamics:

$$
\mathrm{d} Q_{\mathrm{t}}=\mathrm{d} H_{\mathrm{t}}-\mathrm{d} W_{\mathrm{t}}=\mathrm{d} U_{\mathrm{t}}+\mathrm{d} E_{\text {kin }, t}-\mathrm{d} W_{\mathrm{t}}
$$

where $\mathrm{d} U_{\mathrm{t}}=(1 / 2)\left(\kappa_{t+\Delta t} x_{t+\Delta t}^{2}-\kappa_{\mathrm{t}} x_{\mathrm{t}}^{2}\right)$ is the change in potential energy, $\mathrm{d} E_{\mathrm{kin}, t}=(m / 2)\left(v_{\mathrm{t}+\Delta t}^{2}-v_{\mathrm{t}}^{2}\right)$ is the kinetic energy change, and $\mathrm{d} W_{\mathrm{t}}$ is given by equation (2). Equation (3) equals Sekimoto's celebrated expression for microscopic heat ${ }^{6}$. Both work and heat along the elementary processes in the cycle are calculated by summing the contributions of equations (2) and (3) from the beginning to the end of the process.

The kinetic energy of the particle (of mass $m$ and friction coefficient $\gamma$ ) at time $t, v_{\mathrm{t}}$, is calculated from the time-averaged velocity at time $t, \bar{v}_{\mathrm{t}}=\left(x_{\mathrm{t}+\Delta t}-x_{\mathrm{t}}\right) / \Delta t$ :

$$
v_{\mathrm{t}}^{2}=\mathcal{L}_{\mathrm{t}} \bar{v}_{\mathrm{t}}^{2}
$$

where $\mathcal{L}_{\mathrm{t}}$ is a correction factor that depends on the acquisition frequency $f=1 / \Delta t$ and on the physical parameters of the system at time $t$ :

$$
\mathcal{L}_{\mathrm{t}}=\frac{1}{2 f^{2}}\left[\frac{1}{f_{0}^{2}}+\frac{\mathrm{e}^{-\frac{f_{\mathrm{p}}}{2 f}}}{f_{1}}\left(\frac{\mathrm{e}^{-f_{1} / f}}{f_{\mathrm{p}}+2 f_{1}}-\frac{\mathrm{e}^{f_{1} / f}}{f_{\mathrm{p}}-2 f_{1}}\right)\right]^{-1}
$$

where $f_{\mathrm{p}}=\gamma / m, f_{\kappa}=\kappa_{\mathrm{t}} / 2 \pi \gamma, f_{0}=\sqrt{f_{\mathrm{p}} f_{\kappa}}$ and $f_{1}=\sqrt{f_{\mathrm{p}}^{2} / 4-f_{0}^{2}}($ refs 13,14).
The Shannon entropy of the particle at time $t, S_{\mathrm{t}}$, is measured as the sum of the positional and kinetic entropy, $S_{\mathrm{t}}=S_{x, \mathrm{t}}+S_{v, \mathrm{t}}$. Here, $S_{x, \mathrm{t}}=-k \int \mathrm{d} x_{\mathrm{t}} \rho\left(x_{\mathrm{t}}, t\right) \ln \rho\left(x_{\mathrm{t}}, t\right)$, where $\rho\left(x_{\mathrm{t}}, t\right)$ is the PDF to observe trajectories that pass through $x_{\mathrm{t}}$ at time $t$ along the experiment. The distribution $\rho\left(x_{\mathrm{t}}, t\right)$ is estimated from the histogram of $x_{\mathrm{t}}$ using a regular binning of 25 bins in the interval $\left[-3 \sigma\left(x_{\mathrm{t}}\right), 3 \sigma\left(x_{\mathrm{t}}\right)\right]$, with $\sigma\left(x_{\mathrm{t}}\right)$ being the standard deviation of the position at time $t$. The same procedure is applied to determine $S_{v, \mathrm{t}}=-k \int \mathrm{d} v_{\mathrm{t}} \rho\left(v_{\mathrm{t}}, t\right) \ln \rho\left(v_{\mathrm{t}}, t\right)$, binning in the interval $\left[-3 \sigma\left(v_{\mathrm{t}}\right), 3 \sigma\left(v_{\mathrm{t}}\right)\right]$ in this case, with $v_{\mathrm{t}}$ being determined using equations (4) and (5).

For a given cycle of duration $\tau$, we first calculate the distribution of $\eta_{\tau}^{(\mathrm{i})}$ for $i=[1,2,3, \ldots, 400]$ cycles. For each value of $i$, the $\operatorname{PDF} \rho\left(\eta^{(\mathrm{i})}\right)$ is estimated as follows: we first compute the values of $\eta_{\tau}^{(\mathrm{i})}$ (equation (1) in the main text) along the experiment as the ratio of the work summed over $i$ consecutive cycles over the heat summed over the same $i$ cycles. This procedure is repeated along all of the cycles of the experiment. The distributions shown in Fig. 3 are calculated using a kernel density routine in MATLAB R2013a by partitioning the data in regular bins ranging from $-5 \eta_{\mathrm{C}}$ to $5 \eta_{\mathrm{C}}$ of width $0.02 \eta_{\mathrm{C}}$. The tails of the distribution (inset of Fig. 3) are sampled using a binning of width $0.4 \eta_{\mathrm{C}}$.

Efficiency fluctuations in the quasistatic limit. The traditional definition of efficiency for a heat engine operating between two thermal baths is the ratio between work extracted in the cycle and heat absorbed during the hot isothermal,

$$
\eta_{\tau}^{1,(i)}=\frac{-W_{\tau}^{(i)}}{Q_{3, \tau}^{(i)}}
$$

If heat is exchanged during other steps different from isothermals, even if only because of fluctuations, it may be relevant to reflect this in the efficiency. Thus, these two other definitions for the efficiency are possible:

$$
\eta_{\tau}^{2,(i)}=\frac{-W_{\tau}^{(i)}}{Q_{2, \tau}^{(i)}+Q_{3, \tau}^{(i)}}, \quad \eta_{\tau}^{3,(i)}=\frac{-W_{\tau}^{(i)}}{Q_{2, \tau}^{(i)}+Q_{3, \tau}^{(i)}+Q_{4, \tau}^{(i)}}
$$

For example, $\eta_{\tau}^{2,(i)}$ is the type of efficiency usually considered when the heat in step 2 (the step just before the hot isothermal expansion) is exchanged with the hot bath, as in the microscopic Stirling motor ${ }^{3}$ (with step 2 corresponding to the isochoric heating in this case).

The corresponding long-term efficiencies are obtained with the limits $i \rightarrow \infty$ and $\tau \rightarrow \infty$ :

$$
\begin{gathered}
\eta_{1}=\frac{-W_{\infty}}{Q_{3, \infty}} \\
\eta_{2}=\frac{-W_{\infty}}{Q_{2, \infty}+Q_{3, \infty}} \\
\eta_{3}=\frac{-W_{\infty}}{Q_{2, \infty}+Q_{3, \infty}+Q_{4, \infty}}
\end{gathered}
$$

where $W_{\infty}=\lim _{i \rightarrow \infty}\left(W_{\infty}^{(\mathrm{i})} / i\right)$, and the same for the heat.

Theoretically, these three definitions of long-term efficiency converge to Carnot efficiency, as heat exchanges in the adiabatic steps vanish on average. However, the experimental long-term efficiencies do not coincide for large $\tau$ (data not shown). Carnot efficiency is approached best by $\eta_{3}$ in the quasistatic limit. This can be understood by noting that $\eta_{3}$ is the quantity with the smallest fluctuations around $\eta_{\mathrm{C}}$ of the three in this limit. Thus, when averaging over a finite number of cycles this approaches Carnot efficiency faster.

We can analyse the fluctuations of $\eta_{3}$ in the quasistatic limit by noting that the work is delta distributed. Then, the denominator in $\eta_{3}$ can be expressed using the first law applied to the process $2 \rightarrow 3 \rightarrow 4$, as

$$
Q_{2}+Q_{3}+Q_{4}=H_{I}-H_{\mathrm{II}}-W+W_{1}
$$

where $W$ is the total work in the cycle and $W_{1}$ is work in the cold isothermal, both deterministic quantities. Here I and II refer to the initial and final state of the system during the subprocess 1 . This yields

$$
\eta_{3}=\frac{-W}{-W+W_{1}+H_{\mathrm{I}}-H_{\mathrm{II}}}
$$

where $H_{\mathrm{II}}-H_{\mathrm{I}}$ is the total energy difference between the initial and final state in the cold isothermal and is the only fluctuating quantity in the expression. The quasistatic averages of the work in the cold isothermal and in the cycle are equal to

$$
\begin{gathered}
W=\frac{k}{2}\left(T_{\mathrm{c}}-T_{\mathrm{h}}\right) \log \frac{\kappa_{\mathrm{II}}}{\kappa_{\mathrm{I}}} \\
W_{1}=\frac{k T_{\mathrm{c}}}{2} \log \frac{\kappa_{\mathrm{II}}}{\kappa_{\mathrm{I}}}
\end{gathered}
$$


and then

$$
\eta_{3}=\frac{\eta_{\mathrm{C}}}{1+\frac{2\left(H_{1}-H_{I I}\right)}{k T_{\mathrm{h}} \log \frac{\kappa_{I I I}}{k_{1}}}}
$$

The distribution of the internal energy change is given by

$$
\rho\left(H_{\mathrm{I}}-H_{\mathrm{II}}\right)=\beta_{\mathrm{c}} \mathrm{e}^{-\beta_{\mathrm{c}}\left|H_{\mathrm{I}}-H_{\mathrm{II}}\right|}
$$

with $\beta_{\mathrm{c}}=1 / k T_{\mathrm{c}}$. From this, we can compute the variance of $H_{\mathrm{I}}-H_{\mathrm{II}}$, which reads

$$
\Delta\left(H_{\mathrm{I}}-H_{\mathrm{II}}\right)=\sqrt{2} k T_{\mathrm{c}}
$$

This gives a variance for $\eta_{3}$ of

$$
\Delta \eta_{3}=\eta_{\mathrm{C}}\left|\frac{2}{k T_{\mathrm{h}} \log \frac{\kappa_{\mathrm{II}}}{\kappa_{\mathrm{I}}}}\right| \Delta\left(H_{\mathrm{I}}-H_{\mathrm{II}}\right)
$$

and for the relative fluctuation

$$
\frac{\Delta \eta_{3}}{\eta_{\mathrm{C}}}=\left|\frac{2 \sqrt{2}}{k T_{\mathrm{h}} \log \frac{\kappa_{\mathrm{II}}}{\kappa_{\mathrm{I}}}}\right| k T_{\mathrm{c}}
$$

Equivalently, the variance of $\eta_{1}$ in the quasistatic limit reads

$$
\frac{\Delta \eta_{1}}{\eta_{\mathrm{C}}}=\left|\frac{2 \sqrt{2}}{k T_{\mathrm{h}} \log \frac{\kappa_{\mathrm{II}}}{\kappa_{\mathrm{I}}}}\right| k T_{\mathrm{h}}
$$

Fluctuations on $\eta_{1}$ are a factor $T_{\mathrm{h}} / T_{\mathrm{c}}$ stronger than those of $\eta_{3}$ and values for $\eta_{1}$ computed from averages of a small finite number of cycles will in general be less reliable than those of $\eta_{3}$. When considering the intermediate case of $\eta_{2}$, the variance can be shown to be equal to

$$
\frac{\Delta \eta_{2}}{\eta_{\mathrm{C}}}=\left|\frac{2 \sqrt{2}}{k T_{\mathrm{h}} \log \frac{\kappa_{\mathrm{II}}}{\kappa_{\mathrm{I}}}}\right| \frac{k}{2} \sqrt{T_{\mathrm{h}}^{2}+T_{\mathrm{c}}^{2}}
$$

Our theoretical results predict that the width of the long-term efficiencies satisfy $\Delta \eta_{3} \lesssim \Delta \eta_{2} \ll \Delta \eta_{1}$, which indicates than when estimating efficiencies $\eta_{3}$ is expected to have the least experimental error associated with fluctuations.

\section{References}

33. Sekimoto, K., Takagi, F. \& Hondou, T. Carnot's cycle for small systems: Irreversibility and cost of operations. Phys. Rev. E 62, 7759-7768 (2000). 